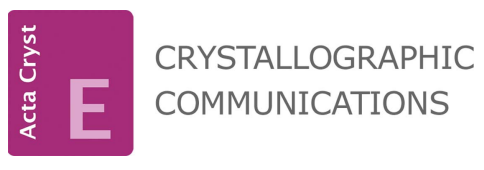

ISSN 2056-9890

Received 17 August 2016

Accepted 26 August 2016

Edited by P. C. Healy, Griffith University, Australia

Keywords: crystal structure; 3-chlorothiophene2-carboxylic acid; 4,4'-bipyridine; O- $\mathrm{H} \cdots \mathrm{N}$ based synthon; co-crystal.

CCDC reference: 1501060

Supporting information: this article has supporting information at journals.iucr.org/e

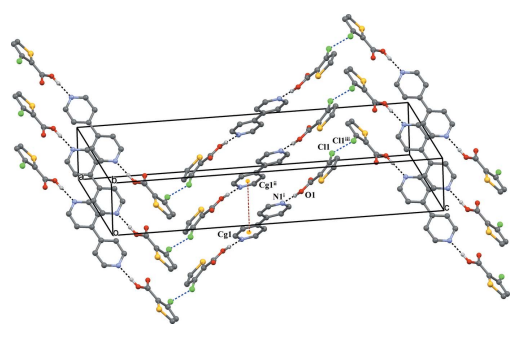

OPEN $\odot$ ACCESS

\section{Supramolecular interactions in the 1:2 co-crystal of 4,4'-bipyridine and 3-chlorothiophene-2-carboxylic acid}

\author{
Olakkandiyil Prajina, ${ }^{\text {a }}$ Packianathan Thomas Muthiah ${ }^{\mathrm{a} *}$ and David K. Geiger ${ }^{\mathrm{b}}$ \\ ${ }^{\mathbf{a}}$ School of Chemistry, Bharathidasan University, Tiruchirappalli 620 024, Tamilnadu, India, and ${ }^{\mathbf{b}}$ Department of \\ Chemistry, SUNY-College at Geneseo, Geneseo, New York 14454, USA. *Correspondence e-mail: \\ tommtrichy@yahoo.co.in
}

The asymmetric unit of the title compound, $2 \mathrm{C}_{5} \mathrm{H}_{3} \mathrm{ClO}_{2} \mathrm{~S} \cdot \mathrm{C}_{10} \mathrm{H}_{8} \mathrm{~N}_{2}$, is comprised of a molecule of 3-chlorothiophene-2-carboxylic acid (3TPC) and half of a molecule of 4,4'-bipyridine (BPY). A distinctive $\mathrm{O}-\mathrm{H} \cdots \mathrm{N}$-based synthon is present. $\mathrm{Cl} \cdots \mathrm{Cl}$ and $\pi-\pi$ stacking interactions further stabilize the crystal structure, forming a two-dimensional network parallel to the $b c$ plane.

\section{Chemical context}

Structurally homogeneous crystalline solids in well defined stochiometry are called co-crystals. In recent years, the physicochemical properties of active pharmaceutical ingredients have been improved widely with the use of co-crystals (Lemmerer \& Bernstein, 2010). Supramolecular synthons - modular representation of primary recognition between functional groups - are of great importance in providing an effective strategy for designing solids in crystal engineering. All geometrical and chemical information of molecular recognition is contained in the structural units called synthons. In the context of co-crystal formation, heterosynthons provide a predictive justification in terms of unique intermolecular interactions (Mukherjee et al., 2011, 2013). There are many literature cases of $\mathrm{O}-\mathrm{H} \cdots \mathrm{N}$-bonded interactions between acid and pyridine-based systems (Shattock et al., 2008; Lemmerer et al., 2015). 4,4'-Bipyridine (BPY) is a weak bidentate base commonly used in crystal engineering on account of its bridging abilities. It also acts as the co-crystal former in the present study because it readily participates in hydrogen bonds with carboxyl-attached organic molecules (Pan et al., 2008).

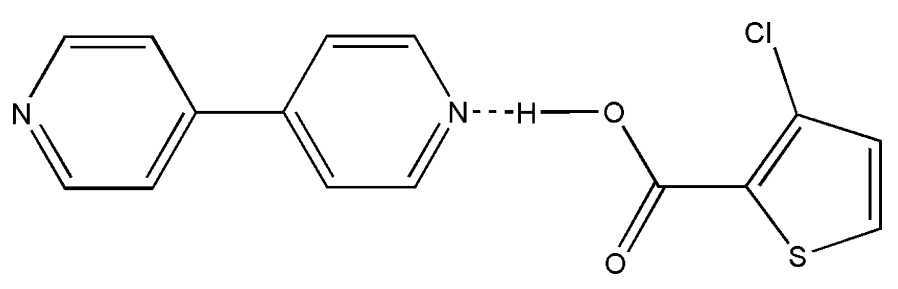

Intermolecular interactions involving halogen substituents, particularly chlorides, play an important role in molecular selfassembly in supra- and biomolecular systems to prepare highly stereoregular organic polymers. It has been observed that these interactions act as a tool in crystal engineering to 


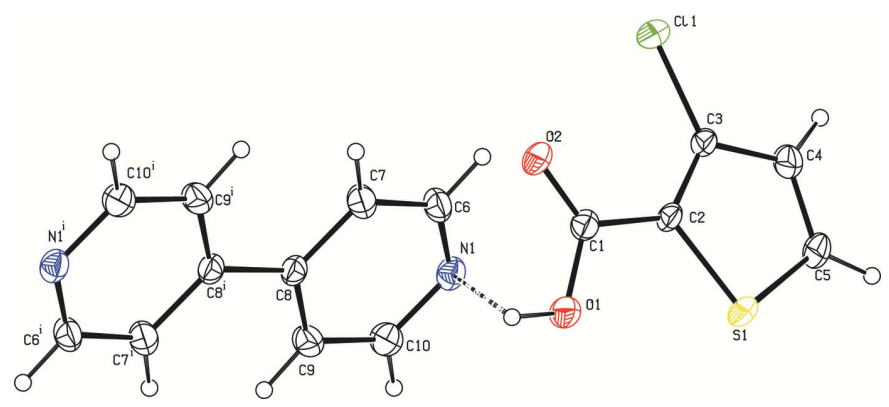

Figure 1

The asymmetric unit of the title compound, showing the atom-numbering scheme. Displacement ellipsoids are drawn at the $30 \%$ probability level. The dashed line represents the $\mathrm{O}-\mathrm{H} \cdots \mathrm{N}$ hydrogen bond. [Symmetry code: (i) $-x+1,-y+1,-z+1$.]

enhance crystal formation and for the design of supramolecular aggregates (Cavallo et al., 2016). In this context, the study of the effect of various halogens on the molecular packing and crystalline architecture of solids has attracted great attention (Csöregh et al., 2001). The structure-forming ability of $\mathrm{Cl} \cdots \mathrm{Cl}$ interactions in assembling chains, ladders, two-dimensional sheets, etc. has been studied extensively (Navon et al., 1997; Metrangolo \& Resnati, 2014). It is based on the values of the two $\mathrm{C}-\mathrm{Hal} \cdots \mathrm{Hal}$ angles, $\theta 1$ and $\theta 2$ (Vener et al., 2013).
Table 1

Hydrogen-bond geometry $\left(\AA,^{\circ}\right)$.

\begin{tabular}{lllll}
\hline$D-\mathrm{H} \cdots A$ & $D-\mathrm{H}$ & $\mathrm{H} \cdots A$ & $D \cdots A$ & $D-\mathrm{H} \cdots A$ \\
\hline $\mathrm{O} 1-\mathrm{H} 1 \cdots \mathrm{N} 1^{\mathrm{i}}$ & $0.89(5)$ & $1.77(5)$ & $2.659(4)$ & $178(5)$ \\
\hline
\end{tabular}

Symmetry code: (i) $x, y-1, z$.

\section{Structural commentary}

The asymmetric unit of the title compound (I) consists of a molecule of 3-chlorothiophene-2-carboxylic acid, 3TPC, and a half of a molecule of 4,4'-bipyridine, BPY, which is located on a crystallographic inversion center. The internal angle at N1 in BPY is $117.1(3)^{\circ}$ and bond lengths [N1-C6= 1.336 (5) $\AA$ and $\mathrm{N} 1-\mathrm{C} 10=1.329$ (5) $\AA$ ] agree with those reported for neutral BPY structures (see for example Jennifer \& Muthiah, 2014; Atria et al., 2014; Moon \& Park, 2012; Qin, 2011; Najafpour et al., 2008). The two external bond angles at the carbon of the carboxyl group are $123.7(3)^{\circ}$ and $112.4(3)^{\circ}$. The high discrepancy between these two angles is typical of an unionized carboxyl group, as are the $\mathrm{C}=\mathrm{O}$ distance of 1.219 (4) $\AA$ and $\mathrm{C}-\mathrm{OH}$ distance of 1.3254 (5) $\AA$ (see for example Prajina et al., 2016; Atria et al., 2014; Jennifer \& Muthiah, 2014; Qin, 2011). The bond distances and angles of the thiophene ring agree with those in structures reported earlier (Zhang et al., 2014).

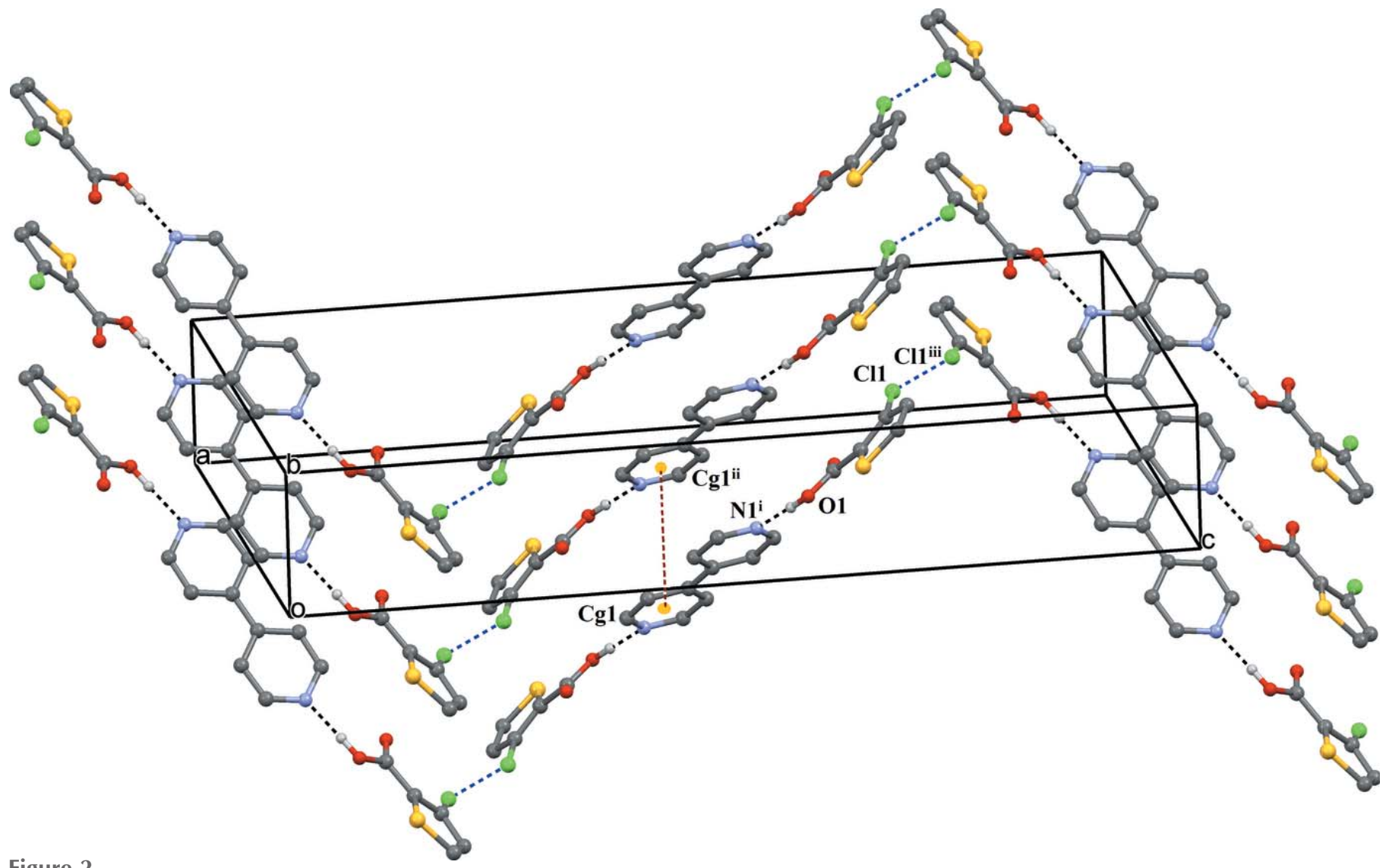

Figure 2

A view of the $\mathrm{O}-\mathrm{H} \cdots \mathrm{N}$ hydrogen bonds (black dashed lines), $\pi-\pi$ stacking (brown dashed lines) and $\mathrm{Cl} \cdots \mathrm{Cl}$ interactions (blue dashed lines). Symmetry codes: (i) $x, y-1, z$; (ii) $1-x, 2-y, 1-z$; (iii) $1-x, y, \frac{1}{2}-z$. 


\section{Supramolecular features}

$3 \mathrm{TPC}$ and BPY are interconnected via $\mathrm{O}-\mathrm{H} \cdots \mathrm{N}$ hydrogenbonding interactions between $(\mathrm{O} 1-\mathrm{H} 1)$ of the carboxyl group and the nitrogen (N1) of BPY (Table 1 and Fig. 1). This $\mathrm{O}-$ $\mathrm{H} \cdots \mathrm{N}$ hydrogen bond is a frequently observed supramolecular synthon in crystal engineering involving a carboxylic acid and a pyridine system (Dubey \& Desiraju, 2015; Lemmerer \& Bernstein, 2010; Mukherjee et al., 2011, 2013; Prajina et al., 2016; Seaton, 2014; Thomas et al., 2010). This supramolecular synthon is also present in the co-crystal of 5-chlorothiophene-2-carboxylic acid with BPY (5TPC44BIPY) and in the co-crystal of thiophene-2-carboxylic acid with BPY reported from our laboratory (Jennifer \& Muthiah, 2014). The co-crystal 5TPC44BIPY and the title co-crystal differ only in the position of chlorine in the thiophene ring with the same base. A chloro derivative was chosen as co-molecule with the expectation that the presence of a $\mathrm{Cl}$ atom would result in halogen-halogen interactions. As expected, a $\mathrm{Cl} \cdots \mathrm{Cl}$ interaction plays the key role in connecting the $\mathrm{O}-\mathrm{H} \cdots \mathrm{N}$ hydrogen-bonded units to form an infinite zigzag chain, i.e., the three-molecule aggregates are further linked to similar neighbouring aggregates through $\mathrm{Cl} \cdots \mathrm{Cl}$ interactions $[3.3925(12) \AA$ 151.71 (1) ${ }^{\circ}$; symmetry code: (iii) $1-x, y, \frac{1}{2}-z$ ] (Vener et al., 2013; Sarma \& Desiraju, 1986; Capdevila-Cortada et al., 2014). The hydrogen-bonded units are stabilized via $\pi-\pi$ stacking interactions between the aromatic systems of BPY molecules $\left[C g 1 \cdots C g 1^{\mathrm{ii}}=3.794\right.$ (2) $\AA$; $C g 1$ is the centroid of the N1/C6/ C7/C8/C9/C10 ring; symmetry code: (ii) $1-x, 2-y, 1-z]$. The perpendicular distance between two parallel molecules is 3.4812 (15) $\AA$. This weak interaction holds the hydrogenbonded chains together, supporting a two-dimensional supramolecular network parallel to the $b c$ plane, as seen in Fig. 2.

\section{Database survey}

In the title compound, the most dominant interaction is the $\mathrm{O}-\mathrm{H} \cdots \mathrm{N}$ hydrogen bond formed between a carboxyl group and a pyridine $\mathrm{N}$ atom (Fig. 1). The length of this hydrogen bond $[\mathrm{O} \cdots \mathrm{N}=2.659$ (4) $\AA$ ] is very close to those of $\mathrm{O}-\mathrm{H} \cdots \mathrm{N}$ bonds found in similar reported co-crystals, such as in the adduct of 2,5-dihydroxy-1,4-benzoquinone and BPY (Cowan et al., 2001) and in the co-crystal of BPY with $N, N^{\prime}$-dioxide-3hydroxy-2-naphthoic acid (1/2) (Lou \& Huang, 2007) and in a series of nine co-crystals involving acridine and benzoic acids (Kowalska et al., 2015). The angle of the hydrogen bond formed between the 3CTPC and BPY molecules is $178(5)^{\circ}$. A similar value is found in the co-crystal of BPY with 3,5-dinitro benzoic acid for which the $\mathrm{O} \cdots \mathrm{N}$ distance is 2.547 (2) $\AA$ (Thomas et al., 2010). In the crystal structure of the co-crystal of adamantane-1,3-dicarboxylic acid and 4,4'-bipyridine, $\pi-\pi$ interactions connect the $\mathrm{O}-\mathrm{H} \cdots \mathrm{N}$ hydrogen-bonded zigzag chains, supporting a two-dimensional network (Pan et al., 2008).
Table 2

Experimental details.

\begin{tabular}{|c|c|}
\hline \multicolumn{2}{|l|}{ Crystal data } \\
\hline Chemical formula & $2 \mathrm{C}_{5} \mathrm{H}_{3} \mathrm{ClO}_{2} \mathrm{~S} \cdot \mathrm{C}_{10} \mathrm{H}_{8} \mathrm{~N}_{2}$ \\
\hline$M_{\mathrm{r}}$ & 481.35 \\
\hline Crystal system, space group & Monoclinic, $C 2 / c$ \\
\hline Temperature $(\mathrm{K})$ & 200 \\
\hline$a, b, c(\AA)$ & $13.538(4), 5.1230(18), 30.167(10)$ \\
\hline$\beta\left(^{\circ}\right)$ & $95.968(9)$ \\
\hline$V\left(\AA^{3}\right)$ & $2080.8(12)$ \\
\hline$Z$ & 4 \\
\hline Radiation type & Мо $K \alpha$ \\
\hline$\mu\left(\mathrm{mm}^{-1}\right)$ & 0.54 \\
\hline Crystal size $(\mathrm{mm})$ & $0.50 \times 0.50 \times 0.10$ \\
\hline \multicolumn{2}{|l|}{ Data collection } \\
\hline Diffractometer & Bruker $S M A R T \mathrm{X} 2 \mathrm{~S}$ benchtop \\
\hline Absorption correction & $\begin{array}{l}\text { Multi-scan (SADABS; Bruker, } \\
\text { 2013) }\end{array}$ \\
\hline$T_{\min }, T_{\max }$ & $0.69,0.95$ \\
\hline $\begin{array}{l}\text { No. of measured, independent and } \\
\text { observed }[I>2 \sigma(I)] \text { reflections }\end{array}$ & $10427,1907,1563$ \\
\hline$R_{\text {int }}$ & 0.078 \\
\hline$(\sin \theta / \lambda)_{\max }\left(\AA^{-1}\right)$ & 0.607 \\
\hline \multicolumn{2}{|l|}{ Refinement } \\
\hline$R\left[F^{2}>2 \sigma\left(F^{2}\right)\right], w R\left(F^{2}\right), S$ & $0.055,0.136,1.19$ \\
\hline No. of reflections & 1907 \\
\hline No. of parameters & 140 \\
\hline $\mathrm{H}$-atom treatment & $\begin{array}{l}\mathrm{H} \text { atoms treated by a mixture of } \\
\text { independent and constrained } \\
\text { refinement }\end{array}$ \\
\hline$\Delta \rho_{\max }, \Delta \rho_{\min }\left(\mathrm{e} \AA^{-3}\right)$ & $0.37,-0.32$ \\
\hline
\end{tabular}

Computer programs: APEX2 (Bruker, 2013), SAINT (Bruker, 2013), SHELXS97 (Sheldrick, 2008), SHELXL2014 (Sheldrick, 2015), PLATON (Spek, 2009), Mercury (Macrae et al., 2008) and publCIF (Westrip, 2010).

\section{Synthesis and crystallization}

To $10 \mathrm{ml}$ of a hot methanol solution of 3TPC (40.6 mg, $25 \mathrm{mmol}), 10 \mathrm{ml}$ of a hot methanolic solution of BPY $(39.0 \mathrm{mg}$, $25 \mathrm{mmol}$ ) was added. The resulting solution was warmed over a water bath for half an hour and then kept at room temperature for crystallization. After a week, clear yellow plates were obtained. The crystal used for X-ray diffraction data collection was cut from a larger crystal.

\section{Refinement}

Crystal data, data collection and structure refinement details are summarized in Table 2. All hydrogen atoms were located in difference Fourier maps. The hydrogen atoms bonded to carbon were refined using a riding model with $\mathrm{C}-\mathrm{H}=0.95 \AA$ and $U_{\text {iso }}(\mathrm{H})=1.2 U_{\text {eq }}(\mathrm{C})$. The carboxylic acid hydrogen atom was freely refined, including its isotropic displacement parameter.

\section{Acknowledgements}

OKP thanks the UGC-SAP and UGC-BSR India for the award of an RFSMS. PTM is thankful to the UGC, New Delhi, for a UGC-BSR one-time grant to Faculty. DKG thanks the US Department of Education for the X-ray diffractometer (grant No. P116Z100020). 


\section{References}

Atria, A. M., Garland, M. T. \& Baggio, R. (2014). Acta Cryst. E70, 157-160.

Bruker (2013). APEX2, SAINT and SADABS. Bruker AXS Inc., Madison, Wisconsin, USA.

Capdevila-Cortada, M., Castelló, J. \& Novoa, J. J. (2014). CrystEngComm, 16, 8232-8242.

Cavallo, G., Metrangolo, P., Milani, R., Pilati, T., Priimagi, A., Resnati, G. \& Terraneo, G. (2016). Chem. Rev. 116, 2478-2601.

Cowan, J. A., Howard, J. A. K. \& Leech, M. A. (2001). Acta Cryst. C57, 302-303.

Csöregh, I., Brehmer, T., Bombicz, P. \& Weber, E. (2001). Cryst. Eng. 4, 343-357.

Dubey, R. \& Desiraju, G. R. (2015). IUCrJ, 2, 402-408.

Jennifer, S. J. \& Muthiah, P. T. (2014). Chem. Cent. J. 8, 20.

Kowalska, K., Trzybiński, D. \& Sikorski, A. (2015). CrystEngComm, 17, 7199-7212.

Lemmerer, A. \& Bernstein, J. (2010). CrystEngComm, 12, 2029-2033.

Lemmerer, A., Govindraju, S., Johnston, M., Motloung, X. \& Savig, K. L. (2015). CrystEngComm, 17, 3591-3595.

Lou, B.-Y. \& Huang, Y.-B. (2007). Acta Cryst. C63, o246-o248.

Macrae, C. F., Bruno, I. J., Chisholm, J. A., Edgington, P. R., McCabe, P., Pidcock, E., Rodriguez-Monge, L., Taylor, R., van de Streek, J. \& Wood, P. A. (2008). J. Appl. Cryst. 41, 466-470.

Metrangolo, P. \& Resnati, G. (2014). IUCrJ, 1, 5-7.

Moon, S.-H. \& Park, K.-M. (2012). Acta Cryst. E68, o1201.
Mukherjee, A., Tothadi, S., Chakraborty, S., Ganguly, S. \& Desiraju, G. R. (2011). Cryst. Growth Des. 11, 2637-2653.

Mukherjee, A., Tothadi, S., Chakraborty, S., Ganguly, S. \& Desiraju, G. R. (2013). CrystEngComm, 15, 4640-4654.

Najafpour, M. M., Hołyńska, M. \& Lis, T. (2008). Acta Cryst. E64, o985.

Navon, O., Bernstein, J. \& Khodorkovsky, V. (1997). Angew. Chem. Int. Ed. Engl. 36, 601-603.

Pan, Y., Li, K., Bi, W. \& Li, J. (2008). Acta Cryst. C64, o41-o43.

Prajina, O., Thomas Muthiah, P. \& Perdih, F. (2016). Acta Cryst. E72, 659-662.

Qin, J.-L. (2011). Acta Cryst. E67, o589.

Sarma, J. A. R. P. \& Desiraju, G. R. (1986). Acc. Chem. Res. 19, 222 228.

Seaton, C. C. (2014). CrystEngComm, 16, 5878-5886.

Shattock, T. R., Arora, K. K., Vishweshwar, P. \& Zaworotko, M. J. (2008). Cryst. Growth Des. 8, 4533-4545.

Sheldrick, G. M. (2008). Acta Cryst. A64, 112-122.

Sheldrick, G. M. (2015). Acta Cryst. C71, 3-8.

Spek, A. L. (2009). Acta Cryst. D65, 148-155.

Thomas, L. H., Blagden, N., Gutmann, M. J., Kallay, A. A., Parkin, A., Seaton, C. C. \& Wilson, C. C. (2010). Cryst. Growth Des. 10, 27702774.

Vener, M. V., Shishkina, A. V., Rykounov, A. A. \& Tsirelson, V. G. (2013). J. Phys. Chem. A, 117, 8459-8467.

Westrip, S. P. (2010). J. Appl. Cryst. 43, 920-925.

Zhang, Q., Luo, J., Ye, L., Wang, H., Huang, B., Zhang, J., Wu, J., Zhang, S. \& Tian, Y. (2014). J. Mol. Struct. 1074, 33-42. 


\section{supporting information}

Acta Cryst. (2016). E72, 1362-1365 [https://doi.org/10.1107/S2056989016013724]

\section{Supramolecular interactions in the 1:2 co-crystal of 4,4'-bipyridine and 3-} chlorothiophene-2-carboxylic acid

\section{Olakkandiyil Prajina, Packianathan Thomas Muthiah and David K. Geiger}

Computing details

Data collection: APEX2 (Bruker, 2013); cell refinement: SAINT (Bruker, 2013); data reduction: SAINT (Bruker, 2013); program(s) used to solve structure: SHELXS97 (Sheldrick, 2008); program(s) used to refine structure: SHELXL2014 (Sheldrick, 2015); molecular graphics: PLATON (Spek, 2009) and Mercury (Macrae et al., 2008); software used to prepare material for publication: publCIF (Westrip, 2010).

bis(3-Chlorothiophene-2-carboxylic acid); 4,4'-bipyridine

\section{Crystal data}

$2 \mathrm{C}_{5} \mathrm{H}_{3} \mathrm{ClO}_{2} \mathrm{~S} \cdot \mathrm{C}_{10} \mathrm{H}_{8} \mathrm{~N}_{2}$

$M_{r}=481.35$

Monoclinic, $C 2 / c$

$a=13.538(4) \AA$

$b=5.1230(18) \AA$

$c=30.167(10) \AA$

$\beta=95.968(9)^{\circ}$

$V=2080.8(12) \AA^{3}$

$Z=4$

\section{Data collection}

Bruker SMART X2S benchtop diffractometer

Radiation source: sealed microfocus tube $\omega$ scans

Absorption correction: multi-scan

(SADABS; Bruker, 2013)

$T_{\min }=0.69, T_{\max }=0.95$

10427 measured reflections

\section{Refinement}

Refinement on $F^{2}$

Least-squares matrix: full

$R\left[F^{2}>2 \sigma\left(F^{2}\right)\right]=0.055$

$w R\left(F^{2}\right)=0.136$

$S=1.19$

1907 reflections

140 parameters

0 restraints
$F(000)=984$

$D_{\mathrm{x}}=1.537 \mathrm{Mg} \mathrm{m}^{-3}$

Mo $K \alpha$ radiation, $\lambda=0.71073 \AA$

Cell parameters from 3438 reflections

$\theta=2.7-24.8^{\circ}$

$\mu=0.54 \mathrm{~mm}^{-1}$

$T=200 \mathrm{~K}$

Plate, clear colourless

$0.50 \times 0.50 \times 0.10 \mathrm{~mm}$

1907 independent reflections

1563 reflections with $I>2 \sigma(I)$

$R_{\text {int }}=0.078$

$\theta_{\max }=25.6^{\circ}, \theta_{\min }=1.4^{\circ}$

$h=-16 \rightarrow 16$

$k=-6 \rightarrow 6$

$l=-36 \rightarrow 36$

Hydrogen site location: mixed

$\mathrm{H}$ atoms treated by a mixture of independent and constrained refinement

$w=1 /\left[\sigma^{2}\left(F_{\mathrm{o}}^{2}\right)+(0.0431 P)^{2}+4.3057 P\right]$ where $P=\left(F_{\mathrm{o}}{ }^{2}+2 F_{\mathrm{c}}{ }^{2}\right) / 3$

$(\Delta / \sigma)_{\max }<0.001$

$\Delta \rho_{\max }=0.37 \mathrm{e} \AA^{-3}$

$\Delta \rho_{\min }=-0.32$ e $\AA^{-3}$ 


\section{Special details}

Geometry. All esds (except the esd in the dihedral angle between two 1.s. planes) are estimated using the full covariance matrix. The cell esds are taken into account individually in the estimation of esds in distances, angles and torsion angles; correlations between esds in cell parameters are only used when they are defined by crystal symmetry. An approximate (isotropic) treatment of cell esds is used for estimating esds involving l.s. planes.

Fractional atomic coordinates and isotropic or equivalent isotropic displacement parameters $\left(\hat{A}^{2}\right)$

\begin{tabular}{lllll}
\hline & $x$ & $y$ & $z$ & $U_{\text {iso }}{ }^{*} / U_{\text {eq }}$ \\
\hline N1 & $0.3544(2)$ & $0.9782(6)$ & $0.54889(10)$ & $0.0363(7)$ \\
C8 & $0.4696(2)$ & $0.6000(7)$ & $0.51024(11)$ & $0.0312(8)$ \\
C7 & $0.4776(3)$ & $0.6415(8)$ & $0.55606(12)$ & $0.0406(9)$ \\
H7 & 0.5227 & 0.5401 & 0.5752 & $0.049^{*}$ \\
C6 & $0.4200(3)$ & $0.8304(8)$ & $0.57370(11)$ & $0.0385(9)$ \\
H6 & 0.4275 & 0.8563 & 0.6051 & $0.046^{*}$ \\
C10 & $0.3468(3)$ & $0.9421(8)$ & $0.50506(12)$ & $0.0423(9)$ \\
H10 & 0.3016 & 1.0481 & 0.4868 & $0.051^{*}$ \\
C9 & $0.4017(3)$ & $0.7571(7)$ & $0.48451(12)$ & $0.0386(9)$ \\
H9 & 0.3931 & 0.7377 & 0.4530 & $0.046^{*}$ \\
S1 & $0.12214(6)$ & $0.6112(2)$ & $0.64475(3)$ & $0.0420(3)$ \\
C11 & $0.40721(6)$ & $0.8001(2)$ & $0.70764(3)$ & $0.0447(3)$ \\
O1 & $0.24836(19)$ & $0.2918(6)$ & $0.59585(9)$ & $0.0462(7)$ \\
O2 & $0.39160(18)$ & $0.3397(5)$ & $0.63983(8)$ & $0.0411(6)$ \\
C2 & $0.2498(2)$ & $0.5841(7)$ & $0.65494(11)$ & $0.0304(7)$ \\
C5 & $0.1177(3)$ & $0.8526(8)$ & $0.68316(12)$ & $0.0454(10)$ \\
H5 & 0.0579 & 0.9341 & 0.6899 & $0.054^{*}$ \\
C4 & $0.2091(3)$ & $0.9160(8)$ & $0.70321(12)$ & $0.0414(9)$ \\
H4 & 0.2210 & 1.0482 & 0.7252 & $0.050^{*}$ \\
C3 & $0.2846(2)$ & $0.7602(7)$ & $0.68722(10)$ & $0.0312(8)$ \\
C1 & $0.3043(3)$ & $0.3936(7)$ & $0.63023(11)$ & $0.0325(8)$ \\
H1 & $0.283(4)$ & $0.184(10)$ & $0.5800(16)$ & $0.072(15)^{*}$ \\
& & & & \\
\hline
\end{tabular}

Atomic displacement parameters $\left(\AA^{2}\right)$

\begin{tabular}{lllllll}
\hline & $U^{11}$ & $U^{22}$ & $U^{33}$ & $U^{12}$ & $U^{13}$ & $U^{23}$ \\
\hline $\mathrm{N} 1$ & $0.0315(16)$ & $0.0350(17)$ & $0.0435(17)$ & $-0.0014(13)$ & $0.0097(13)$ & $-0.0083(13)$ \\
$\mathrm{C} 8$ & $0.0258(18)$ & $0.0349(19)$ & $0.0340(17)$ & $-0.0042(15)$ & $0.0083(14)$ & $-0.0031(14)$ \\
$\mathrm{C} 7$ & $0.041(2)$ & $0.049(2)$ & $0.0321(18)$ & $0.0065(18)$ & $0.0083(16)$ & $-0.0011(16)$ \\
C6 & $0.042(2)$ & $0.042(2)$ & $0.0330(18)$ & $-0.0001(17)$ & $0.0108(15)$ & $-0.0080(15)$ \\
C10 & $0.040(2)$ & $0.046(2)$ & $0.041(2)$ & $0.0100(18)$ & $0.0024(16)$ & $-0.0040(17)$ \\
C9 & $0.041(2)$ & $0.042(2)$ & $0.0331(18)$ & $0.0043(17)$ & $0.0029(15)$ & $-0.0075(15)$ \\
S1 & $0.0207(5)$ & $0.0602(7)$ & $0.0448(5)$ & $0.0069(4)$ & $0.0021(4)$ & $-0.0076(4)$ \\
C11 & $0.0261(5)$ & $0.0630(7)$ & $0.0440(5)$ & $0.0012(4)$ & $-0.0013(4)$ & $-0.0081(4)$ \\
O1 & $0.0313(14)$ & $0.0611(19)$ & $0.0459(15)$ & $0.0083(13)$ & $0.0023(12)$ & $-0.0207(14)$ \\
O2 & $0.0254(13)$ & $0.0515(17)$ & $0.0466(14)$ & $0.0130(12)$ & $0.0047(11)$ & $-0.0053(12)$ \\
C2 & $0.0196(16)$ & $0.040(2)$ & $0.0316(17)$ & $0.0064(14)$ & $0.0048(13)$ & $0.0036(14)$ \\
C5 & $0.031(2)$ & $0.061(3)$ & $0.045(2)$ & $0.0188(19)$ & $0.0092(16)$ & $-0.0057(18)$ \\
C4 & $0.038(2)$ & $0.051(2)$ & $0.0357(19)$ & $0.0114(18)$ & $0.0063(16)$ & $-0.0071(16)$
\end{tabular}




\begin{tabular}{lllllll}
$\mathrm{C} 3$ & $0.0233(17)$ & $0.042(2)$ & $0.0282(16)$ & $0.0065(15)$ & $0.0034(13)$ & $0.0029(14)$ \\
$\mathrm{C} 1$ & $0.0294(19)$ & $0.0352(19)$ & $0.0335(17)$ & $0.0025(15)$ & $0.0056(14)$ & $0.0019(14)$ \\
\hline
\end{tabular}

Geometric parameters $\left(\AA,{ }^{\circ}\right)$

\begin{tabular}{|c|c|c|c|}
\hline $\mathrm{N} 1-\mathrm{C} 10$ & $1.329(5)$ & $\mathrm{S} 1-\mathrm{C} 2$ & $1.729(3)$ \\
\hline $\mathrm{N} 1-\mathrm{C} 6$ & $1.336(5)$ & $\mathrm{C} 11-\mathrm{C} 3$ & $1.721(3)$ \\
\hline $\mathrm{C} 8-\mathrm{C} 7$ & $1.391(5)$ & $\mathrm{O} 1-\mathrm{C} 1$ & $1.325(4)$ \\
\hline $\mathrm{C} 8-\mathrm{C} 9$ & $1.395(5)$ & $\mathrm{O} 1-\mathrm{H} 1$ & $0.89(5)$ \\
\hline $\mathrm{C} 8-\mathrm{C}^{\mathrm{i}}$ & $1.489(7)$ & $\mathrm{O} 2-\mathrm{C} 1$ & $1.219(4)$ \\
\hline $\mathrm{C} 7-\mathrm{C} 6$ & $1.384(5)$ & $\mathrm{C} 2-\mathrm{C} 3$ & $1.374(5)$ \\
\hline $\mathrm{C} 7-\mathrm{H} 7$ & 0.9500 & $\mathrm{C} 2-\mathrm{C} 1$ & $1.472(5)$ \\
\hline $\mathrm{C} 6-\mathrm{H} 6$ & 0.9500 & $\mathrm{C} 5-\mathrm{C} 4$ & $1.359(6)$ \\
\hline $\mathrm{C} 10-\mathrm{C} 9$ & $1.389(5)$ & $\mathrm{C} 5-\mathrm{H} 5$ & 0.9500 \\
\hline $\mathrm{C} 10-\mathrm{H} 10$ & 0.9500 & $\mathrm{C} 4-\mathrm{C} 3$ & $1.421(5)$ \\
\hline $\mathrm{C} 9-\mathrm{H} 9$ & 0.9500 & $\mathrm{C} 4-\mathrm{H} 4$ & 0.9500 \\
\hline $\mathrm{S} 1-\mathrm{C} 5$ & $1.700(4)$ & & \\
\hline $\mathrm{C} 10-\mathrm{N} 1-\mathrm{C} 6$ & $117.1(3)$ & $\mathrm{C} 1-\mathrm{O} 1-\mathrm{H} 1$ & $112(3)$ \\
\hline $\mathrm{C} 7-\mathrm{C} 8-\mathrm{C} 9$ & $116.3(3)$ & $\mathrm{C} 3-\mathrm{C} 2-\mathrm{C} 1$ & $129.8(3)$ \\
\hline $\mathrm{C} 7-\mathrm{C} 8-\mathrm{C} 8^{\mathrm{i}}$ & $121.8(4)$ & $\mathrm{C} 3-\mathrm{C} 2-\mathrm{S} 1$ & $109.7(3)$ \\
\hline $\mathrm{C} 9-\mathrm{C} 8-\mathrm{C}^{\mathrm{i}}$ & $121.8(4)$ & $\mathrm{C} 1-\mathrm{C} 2-\mathrm{S} 1$ & $120.5(3)$ \\
\hline $\mathrm{C} 6-\mathrm{C} 7-\mathrm{C} 8$ & 120.0 & $\mathrm{C} 4-\mathrm{C} 5-\mathrm{S} 1$ & $112.4(3)$ \\
\hline $\mathrm{C} 6-\mathrm{C} 7-\mathrm{H} 7$ & 120.0 & $\mathrm{C} 4-\mathrm{C} 5-\mathrm{H} 5$ & 123.8 \\
\hline $\mathrm{C} 8-\mathrm{C} 7-\mathrm{H} 7$ & 120.0 & $\mathrm{~S} 1-\mathrm{C} 5-\mathrm{H} 5$ & 123.8 \\
\hline $\mathrm{N} 1-\mathrm{C} 6-\mathrm{C} 7$ & $123.4(3)$ & $\mathrm{C} 5-\mathrm{C} 4-\mathrm{C} 3$ & $111.7(3)$ \\
\hline $\mathrm{N} 1-\mathrm{C} 6-\mathrm{H} 6$ & 118.3 & $\mathrm{C} 5-\mathrm{C} 4-\mathrm{H} 4$ & 124.2 \\
\hline $\mathrm{C} 7-\mathrm{C} 6-\mathrm{H} 6$ & 118.3 & $\mathrm{C} 3-\mathrm{C} 4-\mathrm{H} 4$ & 124.2 \\
\hline $\mathrm{N} 1-\mathrm{C} 10-\mathrm{C} 9$ & $123.4(3)$ & $\mathrm{C} 2-\mathrm{C} 3-\mathrm{C} 4$ & $113.8(3)$ \\
\hline $\mathrm{N} 1-\mathrm{C} 10-\mathrm{H} 10$ & 118.3 & $\mathrm{C} 2-\mathrm{C} 3-\mathrm{C} 11$ & $125.4(3)$ \\
\hline $\mathrm{C} 9-\mathrm{C} 10-\mathrm{H} 10$ & 118.3 & $\mathrm{C} 4-\mathrm{C} 3-\mathrm{Cl} 1$ & $120.9(3)$ \\
\hline $\mathrm{C} 10-\mathrm{C} 9-\mathrm{C} 8$ & $119.8(3)$ & $\mathrm{O} 2-\mathrm{C} 1-\mathrm{O} 1$ & $123.9(3)$ \\
\hline $\mathrm{C} 10-\mathrm{C} 9-\mathrm{H} 9$ & 120.1 & $\mathrm{O} 2-\mathrm{C} 1-\mathrm{C} 2$ & $123.7(3)$ \\
\hline $\mathrm{C} 8-\mathrm{C} 9-\mathrm{H} 9$ & 120.1 & $\mathrm{O} 1-\mathrm{C} 1-\mathrm{C} 2$ & $112.4(3)$ \\
\hline $\mathrm{C} 5-\mathrm{S} 1-\mathrm{C} 2$ & $92.46(18)$ & & \\
\hline $\mathrm{C} 9-\mathrm{C} 8-\mathrm{C} 7-\mathrm{C} 6$ & $0.0(5)$ & $\mathrm{S} 1-\mathrm{C} 5-\mathrm{C} 4-\mathrm{C} 3$ & $-0.9(5)$ \\
\hline $\mathrm{C} 8-\mathrm{C} 8-\mathrm{C} 7-\mathrm{C} 6$ & $179.7(4)$ & $\mathrm{C} 1-\mathrm{C} 2-\mathrm{C} 3-\mathrm{C} 4$ & $179.3(3)$ \\
\hline $\mathrm{C} 10-\mathrm{N} 1-\mathrm{C} 6-\mathrm{C} 7$ & $-1.4(6)$ & $\mathrm{S} 1-\mathrm{C} 2-\mathrm{C} 3-\mathrm{C} 4$ & $-0.4(4)$ \\
\hline $\mathrm{C} 8-\mathrm{C} 7-\mathrm{C} 6-\mathrm{N} 1$ & $0.8(6)$ & $\mathrm{C} 1-\mathrm{C} 2-\mathrm{C} 3-\mathrm{Cl} 1$ & $-0.3(6)$ \\
\hline $\mathrm{C} 6-\mathrm{N} 1-\mathrm{C} 10-\mathrm{C} 9$ & $1.4(6)$ & $\mathrm{S} 1-\mathrm{C} 2-\mathrm{C} 3-\mathrm{C} 11$ & $180.0(2)$ \\
\hline $\mathrm{N} 1-\mathrm{C} 10-\mathrm{C} 9-\mathrm{C} 8$ & $-0.7(6)$ & $\mathrm{C} 5-\mathrm{C} 4-\mathrm{C} 3-\mathrm{C} 2$ & $0.9(5)$ \\
\hline $\mathrm{C} 7-\mathrm{C} 8-\mathrm{C} 9-\mathrm{C} 10$ & $-0.1(5)$ & $\mathrm{C} 5-\mathrm{C} 4-\mathrm{C} 3-\mathrm{Cl} 1$ & $-179.5(3)$ \\
\hline $\mathrm{C} 8-\mathrm{C} 8-\mathrm{C} 9-\mathrm{C} 10$ & $-179.7(4)$ & $\mathrm{C} 3-\mathrm{C} 2-\mathrm{C} 1-\mathrm{O} 2$ & $10.6(6)$ \\
\hline $\mathrm{C} 5-\mathrm{S} 1-\mathrm{C} 2-\mathrm{C} 3$ & $-0.1(3)$ & $\mathrm{S} 1-\mathrm{C} 2-\mathrm{C} 1-\mathrm{O} 2$ & $-169.7(3)$ \\
\hline
\end{tabular}




$\begin{array}{llll}\mathrm{C} 5-\mathrm{S} 1-\mathrm{C} 2-\mathrm{C} 1 & -179.8(3) & \mathrm{C} 3-\mathrm{C} 2-\mathrm{C} 1-\mathrm{O} 1 & -168.0(4) \\ \mathrm{C} 2-\mathrm{S} 1-\mathrm{C} 5-\mathrm{C} 4 & 0.6(3) & \mathrm{S} 1-\mathrm{C} 2-\mathrm{C} 1-\mathrm{O} 1 & 11.7(4)\end{array}$

Symmetry code: (i) $-x+1,-y+1,-z+1$.

Hydrogen-bond geometry $\left(\AA,{ }^{\circ}\right)$

\begin{tabular}{lllll}
\hline$D-\mathrm{H} \cdots A$ & $D-\mathrm{H}$ & $\mathrm{H} \cdots A$ & $D \cdots A$ & $D-\mathrm{H} \cdots A$ \\
\hline $\mathrm{O} 1-\mathrm{H} 1 \cdots \mathrm{N} 1^{\mathrm{ii}}$ & $0.89(5)$ & $1.77(5)$ & $2.659(4)$ & $178(5)$ \\
\hline
\end{tabular}

Symmetry code: (ii) $x, y-1, z$. 